\title{
How should we play our hand when holding an ACE?
}

\author{
W. Scott Beattie, MD
}

Published online: 29 May 2010

(C) Canadian Anesthesiologists' Society 2010

Maintenance of blood pressure is controlled in part by the renin angiotensin system (RAS). Angiotensin converting enzyme inhibitors (ACE) and the angiotensin receptor antagonists (ARA) are established as safe and effective antihypertensive agents. When compared with beta blockers, these drugs show equal efficacy in treating blood pressure and they probably have an enhanced safety profile. ${ }^{1,2}$ Furthermore, ACE inhibitors are the agents of choice in hypertensive patients with concomitant diabetes ${ }^{3}$ or compromised left ventricular function. ${ }^{4}$ There is also solid evidence that ACE inhibitors enhance survival in the treatment of vascular disease when added to a regime of beta blockers, statins, and aspirin. ${ }^{5}$ Finally, these agents also improve the quality of life for patients with left ventricular dysfunction. Also, it should be noted that the vast majority of patients on ACE inhibitors are receiving a number of other medications as well.

Therefore, it is not surprising that a large proportion of patients presenting for elective surgery are receiving RAS blockers, either alone or more frequently in combination with another agent, to treat some form of cardiovascular disease. Given the overwhelming evidence favouring RAS blockers in treating cardiovascular disease, it was disconcerting when anecdotal reports arose associating both ACE and ARAs to episodes of severe and intractable hypotension during anesthesia. Perioperative hypotension has been linked to an increased incidence of all forms of adverse cardiac outcomes. ${ }^{6}$ These paradoxical outcomes create a

W. S. Beattie, MD ( $\square)$

Department of Anesthesiology, University Health Network, University of Toronto, Eaton North Wing, 3rd Floor, Room 3-462, 200 Elizabeth Street, Toronto, ON M5G 2C4, Canada e-mail: scott.beattie@uhn.on.ca treatment dilemma. In the perioperative setting, should ACE inhibitors be continued or should they be withheld?

There is limited evidence, i.e., confined to two small randomized controlled trials (RCTs), ${ }^{7,8}$ evaluating the consequences of withholding ACE inhibitors in patients scheduled for elective non-cardiac surgery. These two trials, along with one RCT in cardiac surgery and two observational trials, form the basis of a meta-analysis, suggesting that ACE inhibitors given on the day of surgery increase the incidence of hypotension by $50 \% .^{9}$ However, there was no evidence in this systematic review of any change in the important postoperative outcomes, i.e., stroke, renal failure, myocardial infarction, or death. All of the studies included in this systematic review have methodological problems, including spurious allocation concealment, lack of blinding, and patients lost to followup. Unfortunately, these trials, which were limited to prospective data on 88 vascular surgical patients from one centre, have formed the evidence base for the American College of Physicians' guidelines to withhold ACE inhibitors on the day of surgery. ${ }^{\mathrm{A}}$ There is little evidence to date on the extent to which these guidelines are practiced. In Canada, many anesthesiologists prefer to withhold both ACE inhibitors and ARAs on the day of surgery, while others maintain patients on both groups of medications. Still, others adopt a hybrid position, i.e., withholding ACE inhibitors and ARAs for patients who are receiving multiple antihypertensive medications while giving these drugs on the day of surgery in the subgroup where the only mode of hypertensive control is RAS blockade.

A third observational trial deserves special mention. ${ }^{10}$ Kheterpal et al. analyzed the data from $>65,000$ consecutive patients from 2003 to 2006 . The 9,143 patients who

\footnotetext{
A www.pier.acponline.org.
} 
had RAS inhibition were instructed at three different times to stop these medications on the day of surgery. After propensity matching, ${ }^{\mathrm{B}}$ the results showed that the incidence of hypotension did not differ when comparing a group of patients who had ACE inhibitors withheld and patients who did not receive these agents at the time of the preoperative assessment. In a subgroup of 2,215 patients who were receiving both RAS system blockers and a diuretic, the incidence of hypotension was $4 \%$, a $20 \%$ increase over the control group; however, there was no difference in the incidence of myocardial ischemia or renal failure. The report does suffer from several methodological flaws, most notably, the propensity-matching algorithm failed to adjust for some important confounders. First, the patients receiving ACE inhibitors were, on average, two years older than the ACE inhibitor-naive patients. Second, significantly more patients on ACE inhibitors were receiving beta blockers. Hypotension was observed more often in patients receiving beta blockers perioperatively than in beta blocker-naive patients, and hypotension was associated with adverse outcomes, including death. ${ }^{6,11,12}$ This confounding factor renders Kheterpal et al.'s study difficult to interpret with respect to the management of perioperative non-cardiac surgical patients. All in all, there is simply a paucity of information.

In this issue of the Journal, Railton et al. ${ }^{13}$ add to the body of information with a large retrospective analysis of $>800$ vascular surgery patients. Patients who were receiving ACE inhibitors or ARAs preoperatively were found to have a five-fold increase in all-cause mortality. Is this result biologically plausible? A five-fold increase in mortality is extraordinary; some of the best pharmacologic safety data suggest harm in the order of 20-30\% (not 500\%). How do we reconcile the fact that chronic administration of RAS blockers are associated with a $20 \%$ decrease in adverse events (including death) with a report of a large increase in mortality when these patients come to surgery?

The standardized reporting guidelines recommendations presented in the STROBE statement ${ }^{\mathrm{C}}$ advance the ideal that observational trials must make adjustments to equalize the confounding variables. Since this is an inexact process,

\footnotetext{
${ }^{\mathrm{B}}$ Propensity matching is a tool that can be applied in a variety of fashions yet remains the most sophisticated method of adjusting for confounder imbalance between two cohorts. Propensity scores create a probability (score $0-1$ ) of an event (in this case of receiving reninangiotensin antagonists) and uses this probability to adjust for imbalance. Cohort matching is a separate tool that creates a pair of patients, for example, one taking the drug and one not taking the drug, who have identical probabilities on the basis of propensity scores. If a large population is created by this process, the matching between cohorts approaches that of a randomized trial. However, the probability is based solely on known variables and cannot adjust for unknowns.

C www.strobestatement.org.
}

most observational trials contain the phrase, "causality cannot be inferred; the results are associations only". Paradoxically, every investigator is looking for causality when undertaking an investigation. In the same vein, however, all investigators realize that a true causal relationship can never be elucidated (while some trials approach this goal). All clinical trials have identifiable limitations; all lines of investigation have bias. In the Railton et al. study, every attempt was made to adjust for important confounders using a sophisticated propensitymatching algorithm, and two matched cohorts were created. However, in the case of Railton et al.'s study, it is instructive to examine the important remaining limitations.

Confounding by indication has been described as the most important limitation of observational studies of treatment effects. This type of error is in evidence when prognostic factors influence treatment decisions. In the present study, the ACE inhibitors are the agents of choice in patients who have hypertension with associated diabetes or a history of congestive heart failure $(\mathrm{CHF})$. Both of these co-morbid states are also associated with an increased risk of a poor postoperative outcome. Despite the use of propensity score matching in this report, hypertension and a history of CHF were not well matched. Hypertension was seen more often in the control cohort, which suggests the use of ACE inhibitors was weighted preferentially to patients with coronary disease and CHF. Similar to Kheterpal et al.'s study, ${ }^{10}$ this report found an association between diuretics and adverse outcomes. Railton et al. did not report the types of diuretics. Certainly, the clinical indications for thiazides differ from those for furosemide or aldosterone, the latter being used more frequently in patients with left ventricular dysfunction. The study did not capture objective measures of ventricular function through stress tests, echocardiograms, or effort tolerance. Thus, even after matching, it is entirely possible that patients in the RAS blockade group had less cardiac reserve than the matched set not on ACE inhibitors.

Readers are reminded that the data in this analysis spans an eight-year period, a time frame that saw major changes in both the types of vascular procedures performed and the risk attendant in those procedures. With the expansion of endovascular programs, open repair of aortic aneurysms is less frequent, but open repair confers a four- to five-fold increase in risk. ${ }^{14,15}$ Thus, the risk of open surgery in the later portion of this cohort differs from that at the beginning of the data collection. This change is also confounded by the prevalence of RAS blockade in the same time period. Adjustment was not attempted for either of these confounders.

The incidence of renal failure and postoperative dialysis was $4.5 \%$, which is about five times higher than that reported for other series with supra renal aortic clamping. ${ }^{16}$ 
The data in this report were collected from two teaching hospitals, and the surgical procedures were performed by a number of surgeons. The report did not specify the number of suprarenal procedures or the cross-clamping times. Again, none of these factors was considered in the final model.

Finally, we do not know the number of patients who had ACE inhibitors withdrawn on the day of surgery. An assumption appears to have been made that all cardiac medications were taken in the perioperative period. Is this assumption valid? Until now, our impression of the practice in Canada is one of clinical equipoise.

Clinicians who believe that ACE inhibitors are harmful will use this report to further justify their practice. Conversely, those who continue RAS blockade on the day of surgery are unlikely to change their practice on the basis of this report. Most importantly, this report confirms that there is a paucity of good data on which to base the management of patients with RAS inhibition, and there should be a clarion call for collaboration to gather more and higherquality information.

At this point, with regard to optimizing the perioperative management in patients taking ACE and ARA, no bet is safe.

\section{Comment jouer ses cartes lorsqu'on a un as en main?}

Le maintien de la tension artérielle est en partie contrôlé par le système rénine-angiotensine (SRA). Il a été établi que les inhibiteurs de l'enzyme de conversion de l'angiotensine (IECA) et les antagonistes des récepteurs de l'angiotensine (ARA) sont des agents antihypertenseurs sécuritaires et efficaces. Comparativement aux bêtabloquants, ces médicaments ont une efficacité égale pour le traitement de la tension artérielle et présentent probablement un meilleur profil d'innocuité. ${ }^{1,2}$ De plus, les IECA sont les agents de choix pour les patients hypertendus qui souffrent de diabète ${ }^{3}$ concomitant ou dont la fonction du ventricule gauche est affaiblie. ${ }^{4}$ Des données convaincantes démontrent aussi que les IECA améliorent la survie dans le traitement des maladies vasculaires lorsqu'ils sont ajoutés à un régime de bêtabloquants, de statines et d'aspirine. ${ }^{5}$ Enfin, ces agents améliorent également la qualité de vie des patients souffrant de dysfonctionnement du ventricule gauche. En outre, il est à noter que la plupart des patients suivant un traitement d'IECA reçoivent simultanément plusieurs autres médicaments.

Pour toutes ces raisons, il n'est pas étonnant qu'une vaste proportion de patients devant subir une chirurgie non urgente reçoive des inhibiteurs du SRA, administrés seuls ou souvent en combinaison à un autre agent, afin de traiter une maladie cardiovasculaire. Étant donné les preuves assommantes en faveur de l'utilisation d'inhibiteurs du SRA pour traiter les maladies cardiovasculaires, l'apparition de comptes-rendus anecdotiques associant les IECA et les ARA à des épisodes graves d'hypotension difficiles à traiter pendant l'anesthésie a été pour le moins déconcertante. Un lien a été établi entre l'hypotension périopératoire et une incidence accrue d'événements cardiaques défavorables de toutes sortes. ${ }^{6}$ Ces événements paradoxaux créent un dilemme thérapeutique. Dans le contexte périopératoire, les IECA devraient-ils être maintenus, ou devrait-on les éviter?

Nous ne disposons que de peu de données probantes évaluant les conséquences de la non-administration d'IECA chez des patients devant subir une chirurgie non cardiaque et non urgente; ces données sont tirées de deux petites études randomisées contrôlées (ERC). ${ }^{7,8}$ Ces deux études, outre une ERC en chirurgie cardiaque et deux études observationnelles, forment la base d'une méta-analyse suggérant que l'administration d'IECA le jour de la chirurgie augmente l'incidence d'hypotension de $50 \%$. $^{9}$ Toutefois, cette revue méthodique ne fournit aucune donnée probante concernant un quelconque changement au niveau des événements cliniques postopératoires, soit l'accident vasculaire cérébral (AVC), l'insuffisance rénale, l'infarctus du myocarde et le décès. Toutes les études examinées dans cette revue méthodique comportent des problèmes méthodologiques, notamment une dissimulation de l'attribution douteuse, le manque de dissimulation en insu, et la perte de patients durant le suivi. Malheureusement ces études, qui se limitaient à des données prospectives portant sur 88 patients de chirurgie vasculaire d'un seul centre, ont constitué le fondement des recommandations du Collège américain des médecins (American College of Physicians) concernant le retrait des IECA le jour de la chirurgie. ${ }^{\mathrm{A}}$ À ce jour, peu de données probantes rendent compte de la mesure dans laquelle ces directives sont suivies. Au Canada, nombreux sont les anesthésiologistes qui préfèrent ne pas donner d'IECA ou d'ARA le jour de la chirurgie, alors que d'autres maintiennent l'administration de ces deux groupes de médicaments. D'autres encore adoptent une position intermédiaire: ils ne donnent pas d'IECA ou d'ARA aux patients recevant plusieurs médicaments antihypertenseurs, alors qu'ils les administrent le jour de la chirurgie au sous-groupe de patients pour lequel le seul mode de contrôle de l'hypertension est le bloc du SRA.

Une troisième étude observationnelle mérite une attention particulière $\left[{ }^{10}\right]$. Kheterpal et coll. ont analysé les données de $>65000$ patients consécutifs entre 2003 et

\footnotetext{
${ }^{\text {A }}$ www.pier.acponline.org
} 
2006. On a demandé à trois moments différents aux 9143 patients recevant des inhibiteurs du SRA d'interrompre la prise de ces médicaments le jour de la chirurgie. Après appariement par propension, ${ }^{\mathrm{B}}$ les résultats ont montré qu'il n'y avait pas de différence dans l'incidence d'hypotension lorsqu' on comparait un groupe de patients chez lesquels on avait interrompu l'administration d'IECA à des patients qui ne prenaient pas ces agents au moment de l'évaluation préopératoire. Dans un sous-groupe de 2215 patients recevant des inhibiteurs du SRA et un diurétique, l'incidence d'hypotension était de $4 \%$, une augmentation de $20 \%$ par rapport au groupe témoin; toutefois, aucune différence n'a été observée dans l'incidence d'ischémie myocardique ou d'insuffisance rénale. Ce compte-rendu comporte de nombreuses lacunes méthodologiques, la principale étant que l'algorithme d'appariement par propension ne s'est pas ajusté à certaines variables parasites importantes. Premièrement, les patients recevant des IECA avaient, en moyenne, deux ans de plus que les patients naifs aux IECA. Deuxièmement, un nombre significativement plus élevé de patients recevant des IECA recevait également des bêtabloquants. L'hypotension a été observée plus souvent chez les patients recevant des betabloquants en période périopératoire que chez les patients naifs aux bêtabloquants, et l'hypotension a été associée à des pronostics défavorables, y compris le décès. ${ }^{6,11,12}$ Cette variable parasite rend difficile l'interprétation de l'étude de Kheterpal et coll. en ce qui touche à la prise en charge périopératoire des patients de chirurgie non cardiaque. En somme, nous manquons cruellement de données.

Dans ce numéro du Journal, Railton et coll. ajoutent à ces données grâce à une importante analyse rétrospective portant sur $>800$ patients de chirurgie vasculaire. Une multiplication par cinq de la mortalité, toutes causes confondues, a été observée chez les patients recevant des IECA ou des ARA en période préopératoire. Ce résultat est-il biologiquement raisonnable? Une multiplication par cinq de la mortalité est exceptionnelle; certaines des meilleures données portant sur l'innocuité pharmacologique suggèrent des résultats de l'ordre de 20-30\% (pas $500 \%$ !). Comment réconcilier le fait que

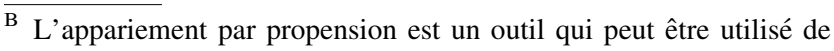
diverses manières tout en demeurant la méthode la plus sophistiquée d'ajustement pour tenir compte de variables parasites entre deux cohortes. Les scores de propension créent une probabilité (score 0-1) d'un événement (dans ce cas le fait de recevoir des antagonistes rénine-angiotensine) et utilisent cette probabilité pour ajuster tout déséquilibre. L'appariement de cohorte est un outil distinct qui crée une paire de patients - par exemple, l'un prenant le médicament et l'autre ne le prenant pas-ayant des probabilités identiques selon les scores de propension. Si une vaste population est ainsi créé, l'appariement entre les cohortes se rapproche à celui d'une étude randomisée. Toutefois, la probabilité est basée exclusivement sur des variables connues et ne peut être ajustée pour des inconnues.
}

l'administration chronique d'inhibiteurs du SRA soit associée à une réduction de $20 \%$ des événements défavorables (y compris la mort) avec l'observation d'une augmentation considérable de la mortalité lorsque ces patients se font opérer?

Selon les directives normalisées de présentation décrites dans l'énoncé STROBE, ${ }^{\mathrm{C}}$ l'idéal serait de procéder à des ajustements dans les études observationnelles pour tenir compte des variables parasites. Étant donné qu'il s'agit là d'un processus inexact, la plupart des études observationnelles mentionnent que «la causalité ne peut être déduite, les résultats ne sont que des associations ». Paradoxalement, tous les chercheurs recherchent la causalité lorsqu'ils entreprennent une étude. En revanche, tous les chercheurs réalisent aussi qu'une véritable relation de causalité ne peut jamais être élucidée (bien que certaines études se rapprochent de cet objectif). Toute étude clinique comporte des lacunes identifiables; tous les domaines de recherche comportent des biais. Dans l'étude de Railton $e t$ coll., les auteurs ont fait tout ce qui était en leur pouvoir pour ajuster leurs données aux variables parasites à l'aide d'un algorithme sophistiqué d'appariement par propension, et deux cohortes appariées ont été créées. Néanmoins, dans le cas de cette étude, l'observation des autres lacunes importantes est édifiante.

Le biais d'indication a été décrit comme étant la lacune la plus importante des études observationnelles sur les effets de traitements. Ce type d'erreur est évident lorsque des facteurs pronostiques influencent les décisions de traitement. Dans l'étude en question ici, les IECA sont les agents privilégiés pour les patients souffrant d'hypertension associée à du diabète ou qui présentent des antécédents d'insuffisance cardiaque congestive (ICC). Ces deux états co-morbides sont également associés à un risque accru de mauvais pronostic postopératoire. Malgré le recours, dans ce compte-rendu, à un système d'appariement par des scores de propension, l'hypertension et les antécédents d'ICC n'ont pas été bien appariés. L'hypertension a été plus souvent observée dans la cohorte témoin, ce qui suggère que l'utilisation d'IECA a été pondérée de façon préférentielle chez les patients souffrant de maladie cardiaque ou d'ICC. Tout comme l'étude de Kheterpal et coll. ${ }^{10}$ ce compte-rendu fait état d'une association entre les diurétiques et un pronostic plus sombre. Railton et coll. ne mentionnent pas le type de diurétiques. Il va sans dire que les indications cliniques pour l'administration de thiazides sont différentes de celles pour le furosémide ou l'aldostérone, cette dernière étant plus fréquemment utilisée chez les patients souffrant de dysfonctionnement du ventricule gauche. L'étude n'a pas identifié de mesures objectives de la fonction ventriculaire à l'aide d'épreuves d'effort,

\footnotetext{
$\overline{\mathrm{C} \text { www.strobestatement.org }}$
} 
d'échocardiogrammes ou de tolérance à l'effort. Dès lors, et ce même après appariement, il est tout à fait possible que les patients du groupe inhibition du SRA disposaient d'une réserve cardiaque moindre que le groupe apparié ne recevant pas d'IECA.

On rappelle aux lecteurs que les données de cette analyse couvrent une période de huit ans, soit une période ayant vu des changements majeurs tant au niveau des interventions vasculaires réalisées qu'à celui des risques attenants à ces interventions. Grâce à l'expansion des programmes endovasculaires, la réparation des anévrismes aortiques par chirurgie ouverte est moins fréquente, mais celle-ci est liée à une multiplication par quatre ou cinq des risques. ${ }^{14,15}$ Ainsi, le risque lié à une chirurgie ouverte dans la portion la plus récente de cette cohorte est différent de celui encouru au début de la récolte de données. À ce changement s'ajoute aussi la variable parasite qu'est la prévalence des blocs du SRA à la même époque. Aucun ajustement n'a été entrepris pour tenir compte de ces deux variables parasites.

L'incidence d'insuffisance rénale et de dialyse postopératoire était de $4,5 \%$, soit environ cinq fois plus que celle rapportée dans d'autres séries portant sur un clampage aortique supra-rénal. ${ }^{16}$ Les données de ce compte-rendu ont été colligées dans deux hôpitaux universitaires, et les interventions chirurgicales ont été réalisées par de nombreux chirurgiens. Le compte-rendu n'a pas spécifié le nombre d'interventions supra-rénales ou les temps de clampage total. Une fois de plus, on n'a tenu compte d'aucun de ces facteurs dans le modèle final.

Enfin, nous ne connaissons pas le nombre de patients chez qui le traitement aux IECA a été interrompu le jour de la chirurgie. Il semble qu'un postulat ait été posé selon lequel tous les médicaments visant le système cardiaque ont été pris pendant la période périopératoire. Ce postulat est-il valable? Jusqu'à présent, l'impression que nous avons de la pratique au Canada est qu'elle est distribuée également.

Les cliniciens qui croient que les IECA sont nocifs utiliseront ce compte-rendu pour justifier leur pratique. En revanche, ceux qui maintiennent l'inhibition du SRA le jour de la chirurgie ne vont probablement pas modifier leur pratique sur la base de ce compte-rendu. Mais le plus important, c'est que ce compte-rendu confirme le manque de données valables sur lesquelles s'appuyer pour prendre en charge les patients sous traitement inhibiteur du SRA. Ce devrait être un signal pour initier davantage de collaboration afin de recueillir plus de renseignements de bonne qualité.

À l'heure actuelle, en ce qui touche à rendre la prise en charge périopératoire optimale des patients prenant des IECA ou des ARA, aucun pari n'est totalement sûr.

\section{Competing interests None declared.}

\section{References}

1. Law MR, Morris JK, Wald NJ. Use of blood pressure lowering drugs in the prevention of cardiovascular disease: meta-analysis of 147 randomised trials in the context of expectations from prospective epidemiological studies. BMJ 2009; 338: b1665.

2. Neal B, MacMahon S, Chapman N, Blood Pressure Lowering Treatment Trialists' Collaboration. Effects of ACE inhibitors, calcium antagonists, and other blood-pressure-lowering drugs: results of prospectively designed overviews of randomised trials. Blood Pressure Lowering Treatment Trialists' Collaboration. Lancet 2000; 356: 1955-64.

3. American Diabetes Association. Hypertension management in adults with diabetes. Diabetes Care 2004; 27(Suppl 1): S65-7.

4. Dagenais GR, Pogue J, Fox K, Simoons ML, Yusuf S. Angiotensin-converting-enzyme inhibitors in stable vascular disease without left ventricular systolic dysfunction or heart failure: a combined analysis of three trials. Lancet 2006; 368: 581-8.

5. Baker WL, Coleman CI, Kluger J, et al. Systematic review: comparative effectiveness of angiotensin-converting enzyme inhibitors or angiotensin II-receptor blockers for ischemic heart disease. Ann Intern Med 2009; 151: 861-71.

6. POISE Study Group, Devereaux PJ, Yang H, Yusuf S, et al. Effects of extended-release metoprolol succinate in patients undergoing non-cardiac surgery (POISE trial): a randomised controlled trial. Lancet 2008; 371: 1839-47.

7. Brabant SM, Bertrand M, Eyraud D, Darmon PL, Coriat P. The hemodynamic effects of anesthetic induction in vascular surgical patients chronically treated with agiotensin 11 receptor antagonists. Anesth Analg 1999; 89: 1388-92.

8. Bertrand M, Godet G, Meersschaert K, Brun I, Salcedo E, Coriat $P$. Should the angiotensin 11 antagonists be discontinued before surgery? Anesth Analg 2001; 92: 26-30.

9. Rosenman DJ, McDonald FS, Ebbert JO, Erwin PJ, LaBella M, Montori $V M$. Clinical consequences of withholding versus administering renin-angiotensin-aldosterone system antagonists in the preoperative period. J Hosp Med 2008; 3: 319-25.

10. Kheterpal S, Khodaparast O, Shanks A, O'Reilly M, Tremper KK. Chronic angiotensin-converting enzyme inhibitor or angiotensin receptor blocker combined with diuretic therapy is associated with increased episodes of hypotensionin noncardiac surgery. J Cardiothorac Vasc Anesth 2008; 22: 180-6.

11. Devereaux PJ, Beattie WS, Choi PT, et al. How strong is the evidence for the use of perioperative beta blockers in non-cardiac surgery? Systematic review and meta-analysis of randomised controlled trials. BMJ 2005; 331: 313-21.

12. Bangalore S, Wetterslev J, Pranesh S, Sawhney S, Gluud C, Messerli FH. Perioperative beta blockers in patients having noncardiac surgery: a meta-analysis. Lancet 2008; 372: 1962-76.

13. Railton CJ, Wolpin J, Lam-McCulloch J, Belo SE. Renin-angiotensin blockade is associated with increased mortality after vascular surgery. Can J Anesth 2010; 57: doi: 10.1007/s12630-010-9330-4.

14. Greenhalgh RM, Brown LC, Kwong GP, Powell JT, Thompson $S G$, EVAR Trial Participants. Comparison of endovascular aneurysm repair with open repair in patients with abdominal aortic aneurysm (EVAR trial 1), 30-day operative mortality results: randomised controlled trial. Lancet 2004; 364: 843-8.

15. EVAR Trial Participants. Endovascular aneurysm repair and outcome in patients unfit for open repair of abdominal aortic aneurysm (EVAR trial 2): randomised controlled trial. Lancet 2005; 365: 2187-92.

16. Chong T, Nguyen L, Owens CD, Conte MS, Belkin M. Suprarenal aortic cross-clamp position: a reappraisal of its effects on outcomes for open abdominal aortic aneurysm repair. J Vasc Surg 2009; 49: 873-80. 